\title{
Helium-filled soap bubbles tracing fidelity in wall-bounded turbulence
}

\author{
David Engler Faleiros ${ }^{1,2}\left[\right.$ (D) Marthijn Tuinstra ${ }^{1} \cdot$ Andrea Sciacchitano $^{2} \cdot$ Fulvio Scarano $^{2}$
}

Received: 13 July 2017 / Revised: 22 January 2018 / Accepted: 23 January 2018 / Published online: 26 February 2018

(c) The Author(s) 2018. This article is an open access publication

\begin{abstract}
The use of helium-filled soap bubbles (HFSB) as flow tracers for particle image velocimetry (PIV) and particle tracking velocimetry (PTV) to measure the properties of turbulent boundary layers is investigated in the velocity range from 30 to $50 \mathrm{~m} / \mathrm{s}$. The experiments correspond to momentum thickness-based Reynolds numbers of 3300 and 5100 . A single bubble generator delivers nearly neutrally buoyant HFSB to seed the air flow developing over the flat plate. The HFSB motion analysis is performed by PTV using single-frame multi-exposure recordings. The measurements yield the local velocity and turbulence statistics. Planar two-component-PIV measurements with micron-sized droplets (DEHS) conducted under the same conditions provide reference data for the quantities of interest. In addition, the behavior of air-filled soap bubbles is studied where the effect of non-neutral buoyancy is more pronounced. The mean velocity profiles as well as the turbulent stresses obtained with HFSB are in good agreement with the flow statistics obtained with DEHS particles. The study illustrates that HFSB tracers can be used to determine the mean velocity and the turbulent fluctuations of turbulent boundary layers above a distance of approximately two bubble diameters from the wall. This work broadens the current range of application of HFSB from external aerodynamics of large-scale-PIV experiments towards wall-bounded turbulence.
\end{abstract}

\section{Introduction}

The use of helium-filled soap bubbles (HFSB) as tracer particles was initially intended for aerodynamic flow visualization, for instance to visualize the flow around a parachute (Pounder 1956). Their use for quantitative measurements had been initially attempted with bubbles of several millimeters. The results discouraged follow-ups due to the difficulties in producing HFSB that could accurately follow the flow (Kerho and Bragg 1994). This issue has been resolved through the reduction of the bubble diameter to the sub-millimeter range (about $0.3 \mathrm{~mm}$ ), and through refining the control of helium and bubble fluid solution (BFS) mass flows to match the bubble density to that of air (Scarano et al. 2015).

The intense light reflection of HFSB makes them very attractive to perform particle image velocimetry (PIV) at large scale, achieving measurement domains of several square meters in confined spaces (Bosbach et al. 2009;

David Engler Faleiros

david.engler.faleiros@nlr.nl

1 Helicopters and Aeroacoustics Department, Netherlands Aerospace Centre (NLR), Emmeloord, The Netherlands

2 Faculty of Aerospace Engineering, Delft University of Technology, Delft, The Netherlands
Kühn et al. 2011). Their use in wind tunnels has been demonstrated more recently investigating Kármán vortices in a cylinder's wake (Scarano et al. 2015) and tip vortices of a wind turbine (Caridi et al. 2016), with measurement volumes of 5 and 12 liters, respectively. Furthermore, HFSB have been employed to measure the three-dimensional velocity and surface pressure field at the wall past a wall-mounted finite cylinder (Schneiders et al. 2016). Although the boundary-layer region was comprised in the latter measurement domain, the turbulent boundary-layer properties have not been ascertained and the feasibility of employing HFSB tracers to measure turbulence characteristics remains to be established.

A factor limiting the use of HFSB to assess the flow properties close to the wall is the much lower spatial concentration during wind tunnel experiments. Experiments by Caridi et al. (2016) and Schneiders et al. (2016) report less than $1 \mathrm{bubble} / \mathrm{cm}^{3}$. A second issue is the size of the tracers that prevents measurements close to the wall. Of no less importance is the question whether HFSB tracers follow the turbulent fluctuations with acceptable fidelity. From experiments in the stagnation region in front of a cylinder, Scarano et al. (2015) estimated a time response of $10-30 \mu$ s for neutrally buoyant bubbles (the same density as air). Studies dealing with HFSB and air-filled soap bubbles (AFSB) immersed 
in isotropic turbulence (grid generated) at low turbulence levels $(\sim 3 \%)$ have concluded that the turbulence intensity is correctly measured with these tracers independently of bubble weight and diameter (Bourgoin et al. 2011). It was found, however, that particle acceleration deviates from that of the flow. The non-isotropic nature of the turbulent fluctuations in shear layers, along with the higher turbulence levels present therein, raises the question on the ability of HFSB to act as ideal flow tracers for the characterization of turbulent boundary layers and separated flows in typical aerodynamic applications.

The present work focuses on the behavior of HFSB along a zero-pressure-gradient turbulent boundary layer. Wind tunnel experiments are conducted at 30 and $50 \mathrm{~m} / \mathrm{s}$, exceeding the maximum speed of $30 \mathrm{~m} / \mathrm{s}$ previously applied in measurements using HFSB. Two-component planar PIV is employed using the conventional micrometric Di-EthylHexyl-Sebacat (DEHS) particles, providing reference values for the mean velocity and turbulent velocity fluctuations. Air-filled soap bubbles are included to investigate the effects of departure from neutral buoyancy. The measurements with AFSB and HFSB are evaluated by particle tracking velocimetry (PTV) and require a large number of recordings (typically $10^{5}$ ) in single-frame multi-exposure mode to compensate for the low particle concentration. The properties of HFSB and AFSB are thoroughly scrutinized by dedicated experiments that deliver their characteristic time response, following the same approach as in Scarano et al. (2015). Finally, the bubbles' ability to follow fluctuations in a turbulent boundary layer is assessed through evaluation of turbulence statistics and turbulent boundary-layer integral properties.

\section{Particle time response}

Because of the particle finite size and difference in density with respect to the fluid, the particle adapts to changes in the flow velocity in a finite time interval. In the Stokes regime, a particle time response can be defined as follows (Adrian and Westerweel 2011):

$\tau_{p}=\frac{|\bar{\rho}| d_{p}^{2}}{18 \nu \emptyset\left(\operatorname{Re}_{p}\right)}$,

in which $\bar{\rho}=\left(\rho_{p}-\rho\right) / \rho, \rho$ being the fluid density and $\rho_{p}$ the particle density, $d_{p}$ the particle diameter, and $v$ is the fluid kinematic viscosity. The term $\emptyset\left(\operatorname{Re}_{p}\right)$ corrects for deviations from the Stokes regime due to particle size (Clift et al. 1978). The latter will be considered of unit order of magnitude and omitted from the discussion in the remainder. In Eq. (1), $\operatorname{Re}_{p}=\left(\left|\vec{V}_{p}\right|-|\vec{V}|\right) d_{p} / v$ is the particle Reynolds number, $\vec{V}_{p}=\left(u_{p}, v_{p}, w_{p}\right)$ being the particle velocity, and $\vec{V}=(u, v, w)$ the fluid velocity.

This lag results in a slip velocity between fluid and particle that can be calculated from the equation of motion for small spherical particles at zero particle Reynolds number (Maxey and Riley 1983). Assuming fluid acceleration to be equal to particle acceleration and neglecting the unsteady forces (Mei 1996) and body forces (gravity is a fraction of fluid acceleration), the slip velocity in a steady flow is calculated as follows (Mei 1996):

$\vec{V}_{p}-\vec{V}=-\frac{d_{p}^{2} \bar{\rho}}{18 v}\left(\vec{V}_{p} \cdot \nabla\right) \vec{V}_{p}$

Therefore, under the above assumptions, the particle time response is an isotropic constant and can be calculated as the ratio of slip velocity and particle acceleration. Considering velocity and acceleration in the streamwise direction, the particle time response is given by the following:

$\tau_{p}=\frac{\left|u-u_{p}\right|}{\left|u_{p} \frac{\partial u_{p}}{\partial x}\right|}$.

If the particle time response is small in comparison to a characteristic time scale of the flow, the particle is able to follow the flow accurately. The Stokes number

$\mathrm{St}=\frac{\tau_{p}}{\tau}$,

is the non-dimensional number used to quantify the tracing fidelity of a particle, where $\tau$ is the characteristic time scale of the flow. In a turbulent boundary layer, the displacement thickness $\delta^{*}$ may be taken as reference length scale for the relevant velocity fluctuations whereby defining the Stokes number as follows:

$\mathrm{St}_{\delta^{*}}=\frac{\tau_{p} U_{\infty}}{\delta^{*}}$,

in which $U_{\infty}$ is the flow free stream velocity. As the Stokes number approaches zero, particles act like ideal flow tracers. The self-similarity of concentration and velocity profiles was found to depend only on $\mathrm{St}_{\delta^{*}}$ and to be independent of Reynolds number based on momentum thickness for $\operatorname{Re}_{\theta}<2500$ (Sardina et al. 2012).

In the case of helium-filled soap bubbles, because of their large size, a short time response is achieved by matching the bubble density to the air density $(\bar{\rho} \approx 0)$.

Given the wide spectrum of velocity fluctuations in the turbulent boundary layer, the unsteady bubble-fluid interaction gives raise to additional forces that may not be negligible and need to be accounted for. From the equation of 
motion of spherical particles (Mei 1996), the ratio between the added-mass force and the quasi-steady Stokes drag in the streamwise direction is as follows:

$\frac{F_{\mathrm{AM}}}{F_{\mathrm{St}}}=\frac{d_{p}^{2}\left(\frac{D u_{p}}{D t}-\frac{D u}{D t}\right)}{36 v\left(u-u_{p}\right)}$.

Considering fluid flow sinusoidal oscillations of angular frequency $\omega$ and assuming small differences between the flow and the particle motion, the slip velocity is of the type $u_{p}-u=A \sin (\omega t)$. Consequently, the difference in acceleration is $D u_{p} / D t-D u / D t=A \omega \cos (\omega t)$. The latter is obtained in the hypothesis that the tracer follows the fluid oscillations frequency, with some extent of damping due to its inertia. Thus, the above ratio can be approximated as follows:

$\frac{F_{\mathrm{AM}}}{F_{\mathrm{St}}}=\frac{d_{p}^{2} \omega}{36 v}$.

The relative effect of the added-mass force increases quadratically with the tracer particle diameter and linearly with the frequency of fluid oscillation. Considering, for instance, a tracer particle of $0.5 \mathrm{~mm}$ diameter in an air flow $\left(v=1.5 \times 10^{-5} \mathrm{~m}^{2} / \mathrm{s}\right)$ with a characteristic frequency $f(\omega=2 \pi f)$ of $100 \mathrm{~Hz}$, then the ratio $F_{\mathrm{AM}} / F_{\mathrm{St}}$ is equal to 0.25 , meaning that the Stokes drag dominates over the added-mass force. Instead, when the flow has a characteristic frequency of $4 \mathrm{kHz}$, which may be the case in a turbulent boundary layer, then the ratio $F_{\mathrm{AM}} / F_{\mathrm{St}}$ becomes of the order of 100 , and the added-mass force dominates the interaction. Notice that, being proportional to the difference in acceleration between particle and fluid, the added-mass force acts in the direction of reducing the difference $\left|D u / D t-D u_{p} / D t\right|$, thus causing the particle to behave more like an ideal tracer.

Similarly, also the time-history force acts to reduce the slip velocity between particle and fluid. A detailed discussion on the time-history force goes beyond the scope of the present work, and is reported in Daitche (2015).
Based on the above, it should be noticed that the condition St $<0.1$ (Samimy and Lele 1991) is sufficient for the good tracing capability of the tracer particles. Instead, when St $>0.1$, if the particle motion is not dominated by the Stokes drag, the effect of unsteady forces should be considered: the particle can still accurately follow the flow if the force is in a frequency range where unsteady forces are sufficiently large, as it will be shown in the following sections.

\section{Experimental setup}

\subsection{Wind tunnel}

The experiments were performed in the small anechoic wind tunnel KAT of the Netherlands Aerospace Centre (NLR). The KAT is an open jet wind tunnel with an area contraction ratio of 10 and an exit cross section of $38.4 \mathrm{~cm} \times 51.2 \mathrm{~cm}$. The test section was enclosed by $90 \mathrm{~cm}$-long end-plates made of wood, with exception of the upper plate, made of Plexiglas to enable optical access. The free stream turbulence intensity of the tunnel ranges from 0.3 to $0.4 \%$ of the free stream velocity, measured from 10 to $70 \mathrm{~m} / \mathrm{s}$.

\subsection{Seeding particles}

The DEHS is generated using an aerosol generator of LaVision that produces particles predominantly below $1 \mu \mathrm{m}$ with a time response of about $2 \mu$ s (Ragni et al. 2011). The particles are introduced into the wind tunnel far upstream of the turbulence screens to guarantee homogenization of the particle distribution.

The bubble generator employed in the experiments is an HFSB-GEN-V11 developed at TU Delft (Fig. 1). The device follows the principle of that developed by Okuno et al. (1993) and is a nozzle that includes three inner coaxial ducts for helium, bubble fluid solution (BFS), and air as driving fluid. The generator is manufactured with 3D printing allowing its miniaturization down to an external diameter of $8 \mathrm{~mm}$. The area contraction ratio from the point of
Fig. 1 Schematic illustration of the bubble generator used in the experiments (left). Shadow visualization of bubbles at the exit of the generator (right) in bubbling (top) and jetting (bottom) regimes
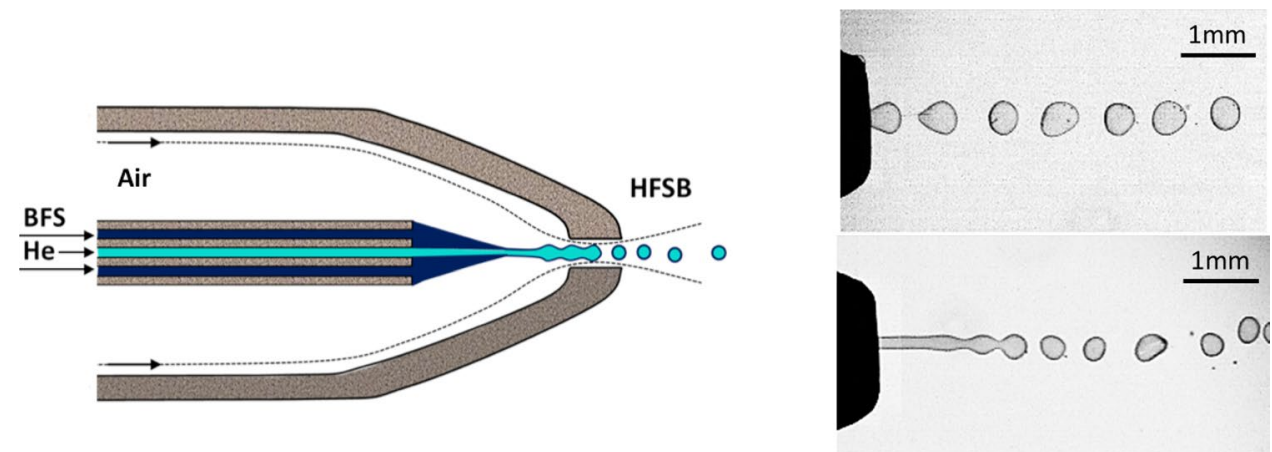
encounter of the three fluids to the orifice nozzle is 19:1. The diameter of the inner tubes and the diameter of the orifice nozzle are 2 and $1 \mathrm{~mm}$, respectively, as in Bosbach et al. (2009). In the standard operations (Table 2), the generator produces between 20,000 and 60,000 bubbles per second. The generator was installed in the settling chamber of the wind tunnel, integrated within a NACA0012-profile wing, with bubbles released at the trailing edge of the airfoil. An in-house fluid supply unit (FSU) was used for the controlled supply of pressurized helium, air, and BFS to the generator. The supply pressure is monitored via manometers installed at the output of each fluid supply line.

\subsection{PIV measurements}

Planar-PIV measurements were conducted using a diode pumped Litron Nd:YLF LDY304 PIV laser $(2 \times 30 \mathrm{~mJ} /$ pulse at $1 \mathrm{kHz}$ ). The images were recorded with a LaVision HighSpeedStar 5 CMOS camera $(1024 \times 1024$ pixels, 12 bits, $20 \mu \mathrm{m}$ pixel pitch). A first experiment was devoted to determine the particle time response and neutral buoyancy condition, measuring the bubbles deceleration in the potential flow upstream of a cylinder, following the approach of Scarano et al. (2015). The conditions of the latter experiment are employed in the measurements of the turbulent boundary-layer properties with HFSB, AFSB, and DEHS as seeding particles.

\subsection{Particle time response}

A cylinder of $50 \mathrm{~mm}$ diameter was installed vertically in the wind tunnel spanning the entire cross section, $15 \mathrm{~cm}$ from the wind tunnel nozzle exit. A $300 \mathrm{~mm}$ splitter plate connected to the cylinder's rear end (Fig. 2) prevents the formation of the von Kármán vortex-street and the consequent unsteady motions that would also be present upstream of the cylinder. The wind tunnel velocity was set to $30 \mathrm{~m} / \mathrm{s}$. The laser was positioned horizontally, perpendicular to the plate. The high-speed camera was above the test section, $65 \mathrm{~cm}$ from the light sheet.

The PIV and PTV recording parameters are summarized in Table 1. PIV measurements using DEHS tracers were made in frame-straddling mode (pulse separation of $30 \mu \mathrm{s}$ ) at $500 \mathrm{~Hz}$. A low acquisition frequency was chosen for faster statistical convergence of the results. In total, a set of 12,000 image pairs was recorded. The PTV recordings using HFSB and AFSB were made in single-frame multi-exposure mode. Images were recorded at a rate of $50 \mathrm{~Hz}$ and the laser at a frequency of $20 \mathrm{kHz}$ (two laser cavities at $10 \mathrm{kHz}$ each, with a time delay of $50 \mu \mathrm{s}$ ), capturing images with full-particle trajectories. This mode of acquisition is possible in the present case where no flow reversal events occur. The exposure time was set to 2.0 and $3.3 \mathrm{~ms}$ for HFSB and AFSB,

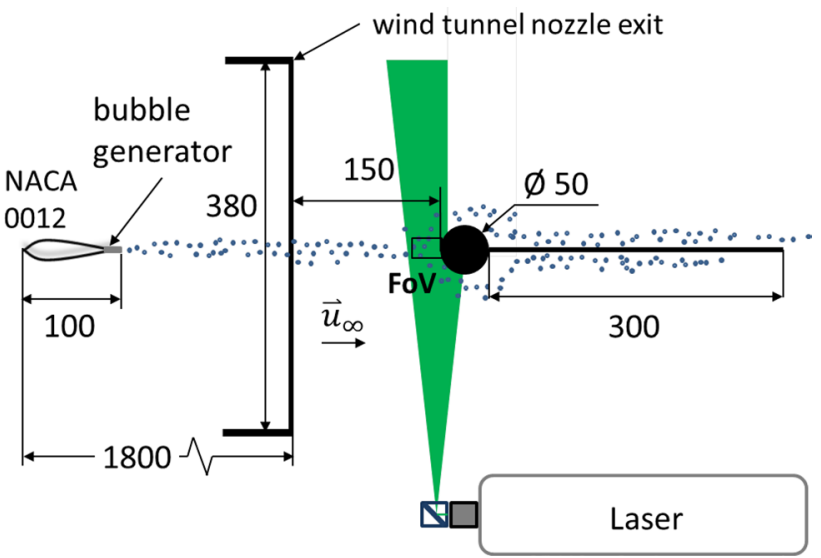

Fig. 2 Top view of experimental setup for cylinder flow. HFSB were not produced during the PIV experiment with DEHS, but the NACA0012 wing was always in place. Dimensions are in millimeters

respectively, to limit the number of trajectories per image. The experiments with bubble tracers yield 5,000 multiply exposed images.

The images from PIV (DEHS) are processed using a spatial cross-correlation algorithm available in LaVision software Davis 8.4. Interrogation windows of $32 \times 32$ pixels with $75 \%$ overlap are used, resulting in a spatial resolution of 2.8 vectors per millimeter. The PTV images are analyzed in Matlab using an in-house tracking algorithm, discussed further in Sect. 4.

The particle tracing fidelity can first be judged qualitatively by comparing the streamlines calculated from the bubbles trajectories to those obtained from PIV measurements using DEHS (Fig. 3). AFSB particles, due to their higher inertia, clearly deviate from the reference along the curved trajectories in front of the cylinder and penetrate closer to the stagnation region before turning sideways. Therefore, the deceleration process is visibly delayed. HFSB feature streamlines that follow closely the reference streamlines (measured with DEHS). Being marginally lighter-than-air in the present case, the streamlines slightly anticipate the air flow deflection.

The particle diameter is estimated from the measured distance between the two glare points $d_{G}$ (Dehaeck et al. 2005). For imaging normal to the illumination direction, the bubble diameter is $\sqrt{2} d_{G}$. The estimated HFSB diameter of $0.55 \mathrm{~mm}$ is somewhat larger than that reported by Caridi et al. (2016) and Scarano et al. (2015) of $0.3 \mathrm{~mm}$. The AFSB were slightly smaller with $0.40 \mathrm{~mm}$ diameter. The bubble size distributions resemble Gaussian distributions (Fig. 4). The polydispersity of the bubble size $\sigma_{d} / d$ is about $10 \%$ for HFSB and $15 \%$ for AFSB.

The above conditions are obtained by carefully controlling that the bubbles are produced in the bubbling regime (Morias et al. 2016) through shadow visualization close to 
Table 1 PIV/PTV recording parameters of cylinder experiment

\begin{tabular}{ll}
\hline Imaging conditions & \\
\hline Camera objective focal length $(\mathrm{mm})$ & 200 \\
Active sensor size $\left(\mathrm{px}^{2}\right)$ & $640 \times 640$ \\
Field of view $\left(\mathrm{mm}^{2}\right)$ & $30 \times 30$ \\
Magnification factor & 0.44 \\
\hline Measurements with HFSB and AFSB (single frame, & multi-exposure) \\
\hline Camera recording frequency & $50 \mathrm{~Hz}$ \\
Laser frequency & $20 \mathrm{kHz}$ \\
Numerical aperture & $f / 32$ \\
Laser sheet thickness (mm) & 5 \\
Exposure time (ms) & $2.0(\mathrm{HFSB}) / 3.3$ \\
& $(\mathrm{AFSB})$ \\
\hline Measurements with DEHS (double frame, single exposure) \\
\hline Double-frame recording frequency & $500 \mathrm{~Hz}$ \\
Pulse separation & $30 \mu \mathrm{s}$ \\
Numerical aperture & $f / 4$ \\
Laser sheet thickness (mm) & 2 \\
Interrogation window (mm) & $1.4(32 \mathrm{px})$ \\
\hline
\end{tabular}

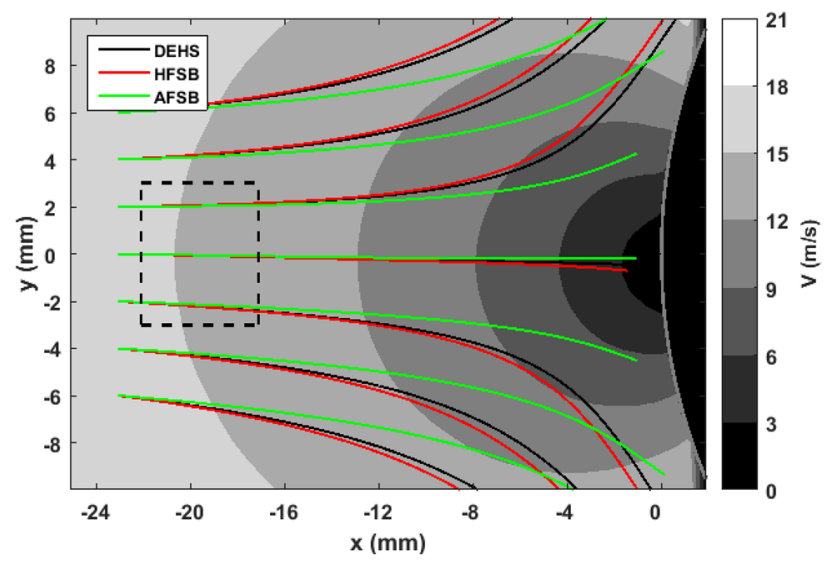

Fig. 3 PIV and PTV measurements of the flow in front of a cylinder. Velocity contours from the DEHS measurements. The dashed square shows the area used to evaluate the time response (see Table 2) the bubble generator exit (Fig. 1). In this regime, the bubbles detach from the cylindrical film region close to the nozzle exit. The resulting distribution is rather monodisperse in terms of bubble diameter as well as for the inferred density, leading to a short response time and a small dispersion of the latter. At higher mass flow rate of helium or air, the production regime was observed to transit to jetting, where the cylindrical soap film region extends for several diameters beyond the orifice exit. Its breakup into bubbles is less regular, yielding a polydisperse distribution of the bubble diameter.

The slip velocity of the bubbles is calculated using DEHS velocity as reference. The particle slip velocity and the particle Lagrangian acceleration are evaluated in a control region as highlighted in Fig. 3. The data closer to the cylinder are not used due to the larger deflection of the streamlines, which would lead to an unfair comparison, since the particles do not follow the exact same trajectories (especially AFSB). The deceleration in this region is about $600 \mathrm{~g}\left(\sim 6000 \mathrm{~m} / \mathrm{s}^{2}\right)$. The ratio between the streamwise components of the slip velocity and the particle acceleration yields the particle time response (Table 2) from (3). The assumption of equal acceleration of particle and fluid is acceptable in such flow (difference is in the order of $5 \%$, Scarano et al. 2015). The ratio of the added-mass force and Stokes drag is estimated from (7) using $f=U_{\infty} / D, D$, being the cylinder diameter. The calculated ratio is about 2 and 1 for HFSB and AFSB, respectively. This indicates that the added-mass force although not dominant is not entirely negligible. In this case, the particle deceleration due to the combined effect of Stokes and added-mass force leads to a slight underestimation of the value of the Stokes time constant $\tau_{p}$. The mean time response of the HFSB is $30 \mu$ s similar to the previous results (Scarano et al. 2015). Because the HFSB tracers are slightly lighter than air, the slip velocity has the same sign of the particle acceleration. The time response distribution of the bubbles is shown in Fig. 5. The value of the time response is multiplied with the sign of the normalized density difference, yielding a negative value for lighter-than-air bubbles. The latter should by no means be interpreted as a negative time
Table 2 AFSB and HFSB volume flow rates, diameter, time response, and density difference

\begin{tabular}{llllllll}
\hline Particle & $Q_{\mathrm{He}}(\mathrm{l} / \mathrm{h})$ & $Q_{\text {air,in }}(\mathrm{l} / \mathrm{h})$ & $Q_{\text {air,ext }}(\mathrm{l} / \mathrm{h})$ & $Q_{\mathrm{BFS}}(\mathrm{ml} / \mathrm{h})$ & $\begin{array}{l}\text { Particle } \\
\text { diameter } d \pm \sigma_{\mathrm{d}} \\
(\mathrm{mm})\end{array}$ & $\begin{array}{l}\text { Time } \\
\text { response, } \\
\tau_{p} \pm \sigma_{\tau}(\mu \mathrm{s})\end{array}$ & $\begin{array}{l}\text { Density, } \\
\rho \pm \sigma_{\bar{\rho}}(\mathrm{kg} / \\
\left.\mathrm{m}^{3}\right)\end{array}$ \\
\hline DEHS & - & - & - & - & $\sim 10^{-3}$ & 2 & 750 \\
$\mathrm{HFSB}$ & 8.8 & - & 53 & 5.5 & $0.55 \pm 0.07$ & $30 \pm 20$ & $1.1 \pm 0.05$ \\
$\mathrm{AFSB}$ & - & 2.4 & 62 & 7.6 & $0.40 \pm 0.06$ & $430 \pm 60$ & $4.4 \pm 0.7$ \\
\hline
\end{tabular}

The standard deviations of the ensemble averages of the particle diameter $\sigma_{\mathrm{d}}$, time response $\sigma_{\mathrm{d}}$, and density difference $\sigma_{\mathrm{d}}$ are also indicated 

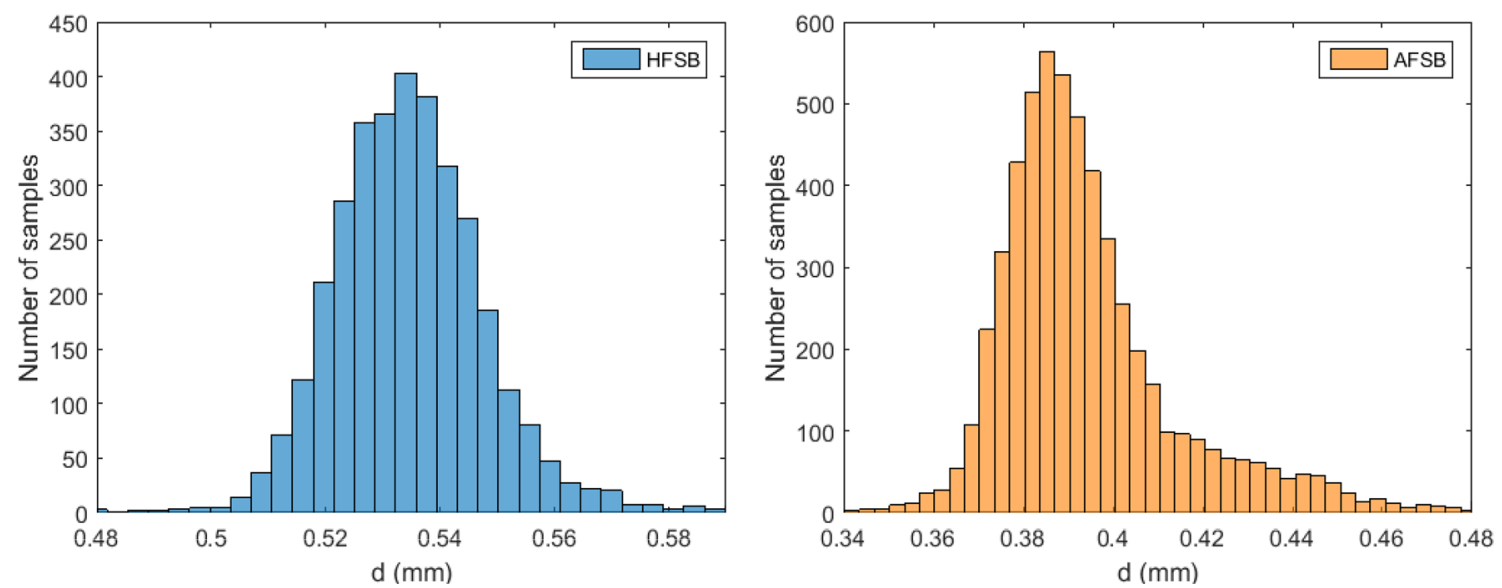

Fig. 4 Size distribution of HFSB and AFSB
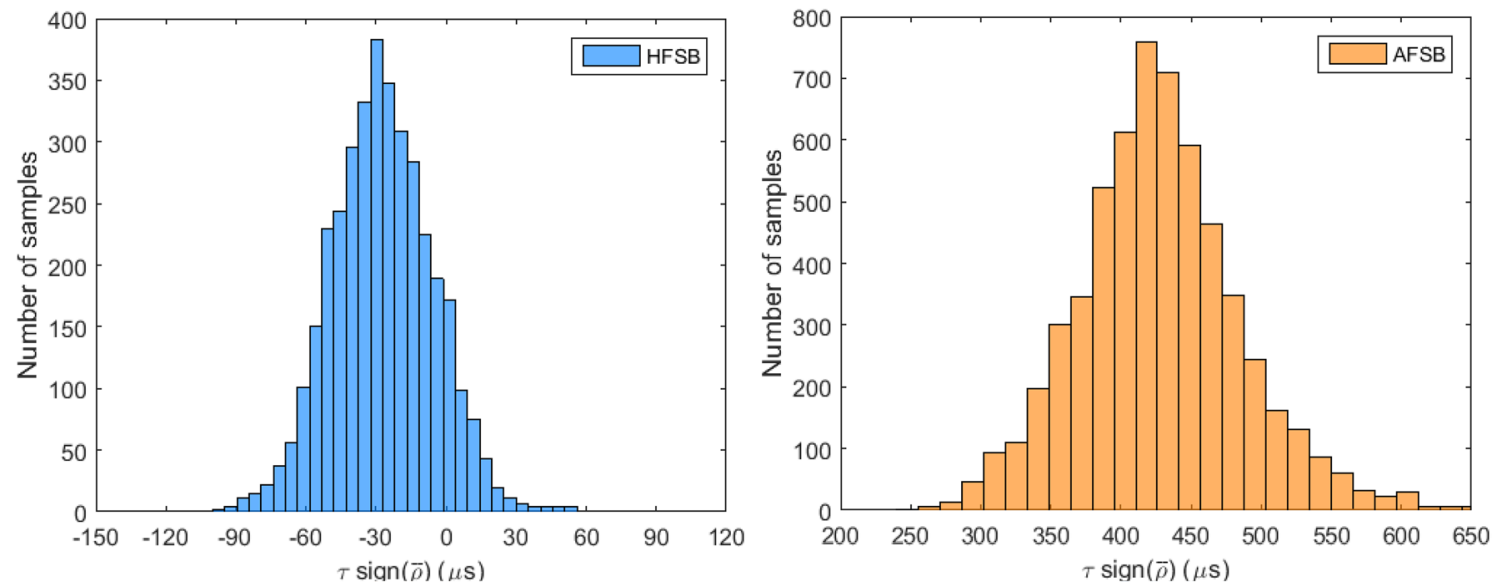

Fig. 5 Time response distribution of HFSB and AFSB

response, but rather indicate that the tracers decelerate earlier than the surrounding air approaching a stagnation point. The time response variation is a consequence of the bubble size distribution together with the variation of the soap film thickness. Both distributions of HFSB and AFSB time responses resemble Gaussian distributions, with the latter showing a broader variation around the mean. The standard deviation of the HFSB time response is about $20 \mu$ s, which is less than previously reported (Morias et al. 2016). The AFSB tracers, although smaller, exhibit a considerably larger mean time response of $430 \mu$ s and standard deviation of $60 \mu \mathrm{s}$, suggesting that a thicker soap film is formed for AFSB.

\subsection{Turbulent boundary-layer measurements}

The turbulent boundary layer developing over a flat plate was measured using the PTV technique to determine the behavior HFSB and AFSB in turbulent flow. The flat plate used is $90 \mathrm{~cm}$ long, $51 \mathrm{~cm}$ wide, and $1 \mathrm{~cm}$ thick with a rounded leading edge of $0.5 \mathrm{~cm}$ radius. The plate was positioned vertically, spanning the entire test section height. A zigzag trip of $1.6 \mathrm{~mm}$ thickness was placed $10 \mathrm{~cm}$ downstream the leading edge forcing the transition to the turbulent flow regime. The experiments were performed at free stream velocity of 30 and $50 \mathrm{~m} / \mathrm{s}$. The experimental setup is shown schematically in Fig. 6.

Experiments conducted with planar PIV and using micron-sized droplets (DEHS) were performed under the same conditions to provide the reference distribution of mean velocity and the turbulence fluctuations statistics. The measurement settings for both PIV and PTV are described in Table 1, with the exception that the PTV measurements were conducted with the camera frequency at $500 \mathrm{~Hz}$ and with $2 \mathrm{~ms}$ exposure time. The measured and inferred properties of the soap bubbles are listed in 
Fig. 6 Top view of the turbulent boundary-layer experiment. No bubbles were produced during the PIV experiment with DEHS, but the NACA0012 wing was always in place. Dimensions are in millimeters

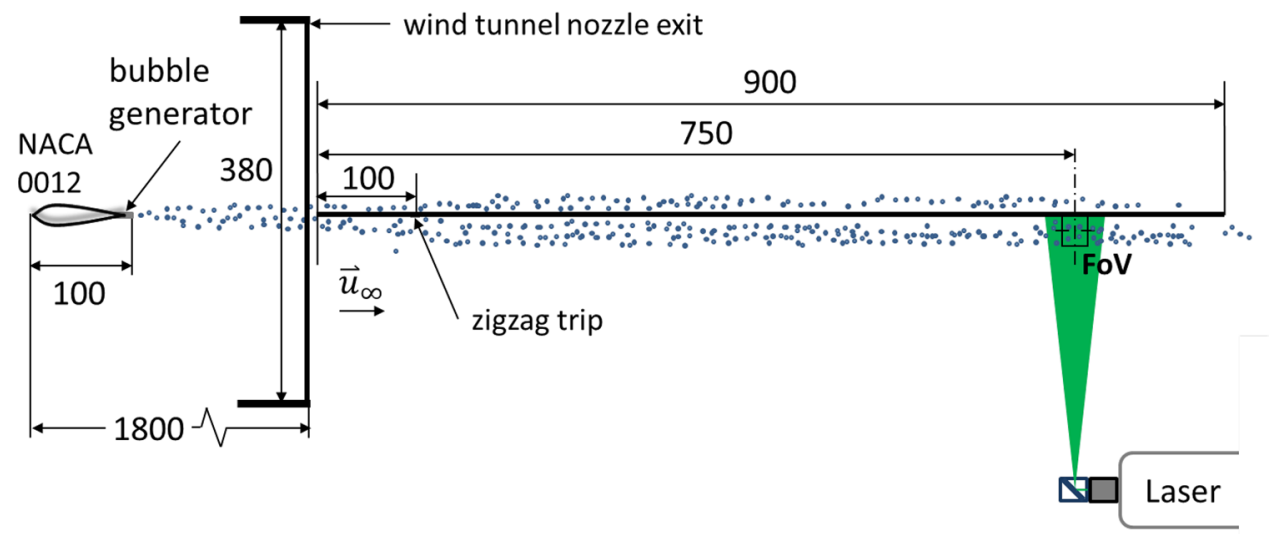

Table 3 Image acquisition for turbulent boundary-layer measurements

\begin{tabular}{lll}
\hline Particle & DEHS & HFSB/AFSB \\
\hline Technique & $\begin{array}{c}\text { Double-frame } \\
\text { single-exposure } \\
\text { PIV }\end{array}$ & $\begin{array}{c}\text { Single-frame } \\
\text { multi-exposure } \\
\text { PTV }\end{array}$ \\
$\begin{array}{ll}\text { Number of runs for each free } \\
\text { stream velocity }\end{array}$ & 5 \\
Number of images per run & 10,000 & 20,000 \\
Total number of images & 10,000 & 100,000 \\
\hline
\end{tabular}

Table 2. For the experiments with bubbles, 100,000 singleframe multi-exposure images were recorded for both bubble types (Table 3 ).

The raw images from PIV (Fig. 7) are processed using spatial cross-correlation algorithm in Davis 8.4. Interrogation windows of $16 \times 16$ pixels with $75 \%$ overlap are used, resulting in 5.5 vectors per millimeter. The PTV data processing is detailed in Sect. 4.

\section{PTV data processing}

The particle tracking analysis is performed using an algorithm based on Malik et al. (1993), combined with a particle displacement predictor that uses the average velocity field from the DEHS data. Only trajectories where the particle image is detected more than five times are considered. A sliding second-order polynomial least-squares fit is applied to the discrete particle positions. The kernels are centered at each particle position, encompassing a minimum of five samples (Fig. 7). If more particle neighbors are available, larger kernels are used up to a maximum of 11 samples. Particle velocity and acceleration are determined from the gradient of the fitted polynomials (Cierpka et al. 2013) at the center of each kernel. The first two and last two vectors in every trajectory are neglected due to the lower accuracy of the polynomial fitting in the trajectory ends. Thus, the smallest acceptable trajectory of only five points would render a single vector. The average number of particles per track after discarding two vectors from each trajectory end is about 20 at $30 \mathrm{~m} / \mathrm{s}$ and 12 at $50 \mathrm{~m} / \mathrm{s}$. The scattered data from the ensemble are averaged
Fig. 7 Left: Raw image from PIV measurements at $U_{\infty}=30 \mathrm{~m} / \mathrm{s}$ (inverted gray scale). Interrogation window is indicated in the image (red square). Mean velocity profile is plotted showing one in every seven vectors for ease of visualization. Right: single-frame multi-exposure PTV images at $U_{\infty}=30 \mathrm{~m} / \mathrm{s}$. Multiple positions of the bubbles are captured along their trajectories. Each bubble produces two glare points with the velocity vector assigned in the middle
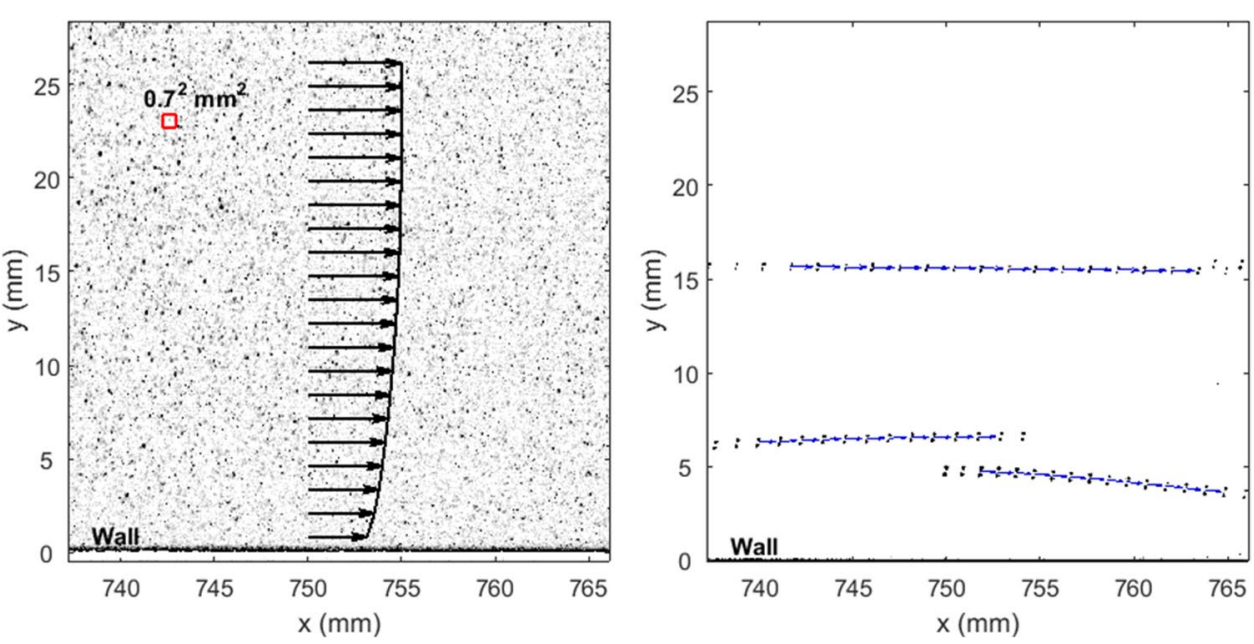
out into bins of $0.9 \times 0.9 \mathrm{~mm}^{2}$ with $50 \%$ overlap, applying radial weighting based on a Gaussian function (Fig. 8). A standard deviation of $0.27 \mathrm{~mm}$ is chosen, so that the weight $w(x, y)$, contributes with 0.5 to a distance of half a grid cell. Every bin encloses a region of the mesh containing $3 \times 3$ grid points to which the Gaussian weighting is applied, resulting in one vector every $0.45 \mathrm{~mm}$. Weighted time-averaged velocity and weighted time-averaged turbulence fluctuations are then calculated for each grid point. The data are further averaged in the streamwise direction along $18 \mathrm{~mm}$. The data points of the HFSB profiles contain on average $3 \times 10^{5}$ vectors at $30 \mathrm{~m} / \mathrm{s}$ and $2 \times 10^{5}$ vectors at $50 \mathrm{~m} / \mathrm{s}$. The concentration of AFSB was, in general, lower, containing on average $2 \times 10^{5}$ and $1 \times 10^{5}$ vectors per data point at 30 and $50 \mathrm{~m} / \mathrm{s}$, respectively. The total processing time is about $0.5 \mathrm{~s}$ per image.

\section{Measurement uncertainty}

The random uncertainty from the reference PIV measurements of the mean velocities, velocity fluctuations, and turbulent stresses is quantified following Sciacchitano and Wieneke (2016). The use of 10,000 image pairs for the reference data guarantees that the mean streamwise velocity and the velocity fluctuations $u_{\text {rms }}^{\prime}$ and $v_{\text {rms }}^{\prime}$ are statistically converged. The uncertainties are computed at 95\% confidence level, assuming a normal error distribution. The random uncertainty of velocity does not exceed 0.2 and $0.1 \%$ of $U_{\infty}$ for the streamwise and wall-normal velocity components, respectively. The uncertainty of the streamwise and wall-normal velocity fluctuations reaches, respectively, a maximum of 2 and $1.5 \%$ of the respective peak values. The uncertainty of the Reynolds shear stress is approximately $5 \%$ of the peak value.

An additional source of uncertainty in this study results from changes of magnification factor across the laser sheet, which was thicker during measurements with bubbles $(5 \mathrm{~mm})$. The variation of optical magnification across

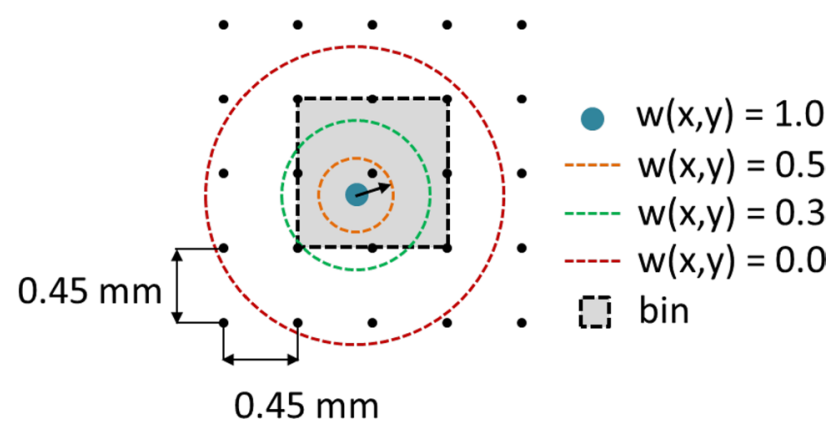

Fig. 8 Radial weighting of scattered data into a grid. Every vector contributes to a bin of $0.9 \mathrm{~mm}^{2}$ the depth from the center plane to the edge of illumination is approximately $0.5 \%$. The latter induces velocity fluctuations of less than $0.5 \%$ with respect to the local velocity measured in the middle plane of the laser sheet. The turbulence intensity measured in the free stream with DEHS is approximately $1 \%$. Therefore, the measurement uncertainty due to magnification changes across the laser sheet can be neglected as it is in the same order of the velocity fluctuations in the free stream.

\section{Boundary-layer properties}

The Reynolds numbers based on momentum thickness are 3300 and 5100 for 30 and $50 \mathrm{~m} / \mathrm{s}$, respectively. The boundary-layer thickness $\delta_{99}$, displacement thickness $\delta^{*}$, momentum thickness $\theta$, and shape factor $\mathrm{H}$ are shown in Table 4. The more robust boundary-layer thickness estimated at $90 \%$ of the free stream velocity $\delta_{90}$ is also included, given the better accuracy of this estimator. A theoretical 1/7 power law profile (Schlichting 1979) is included for reference:

$\delta(x)=0.37 x \mathrm{Re}_{x}^{-1 / 5}, \quad \delta^{*}=\frac{1}{8} \delta, \quad \theta=\frac{7}{72} \delta$.

A comparison of the integral parameters between measured values with DEHS tracers and those predicted from theory generally indicates a good agreement. The boundary-layer thickness $\delta_{99}$ measured with DEHS deviates less than 10 and $20 \%$ from the value obtained from theory for 30 and $50 \mathrm{~m} / \mathrm{s}$, respectively. However, the boundary-layer thickness $\delta_{99}$ measured with DEHS at $50 \mathrm{~m} / \mathrm{s}$ seems to be overestimated, being only $2 \%$ smaller than that measured at $30 \mathrm{~m} / \mathrm{s}$. This difference is about $7 \%$ if $\delta_{90}$ is used for comparison instead, agreeing better with the $10 \%$ difference predicted from theory. Measurements of displacement thickness and momentum thickness with DEHS differ from theoretical values in about $10 \%$. The shape factors exhibit only small deviations of $1 \%$ at $30 \mathrm{~m} / \mathrm{s}$ and $3 \%$ at $50 \mathrm{~m} / \mathrm{s}$. On the above basis, the DEHS data are taken as term of reference to evaluate the measurements conducted with soap bubbles.

The boundary-layer integral properties assessed from HFSB measurements are in sufficient agreement with DEHS. The boundary-layer thickness $\delta_{90}$ is 10 and $13 \%$ larger than DEHS at 30 and $50 \mathrm{~m} / \mathrm{s}$, respectively. The displacement and momentum thicknesses are overestimated by $10 \%$ at $30 \mathrm{~m} / \mathrm{s}$ and $12 \%$ at $50 \mathrm{~m} / \mathrm{s}$. The shape factor deviates less than $0.5 \%$ from the reference also in both cases. Besides, no distinctive differences have been observed between HFSB and AFSB, with almost all the integral values agreeing within $2 \%$. The latter results indicate that the turbulent state of the boundary layer can be reliably established from measurements 
Table 4 Boundary-layer integral values

\begin{tabular}{lllllll}
\hline Particle & $U_{\infty}(\mathrm{m} / \mathrm{s})$ & $\delta_{99}(\mathrm{~mm})$ & $\delta_{90}(\mathrm{~mm})$ & $\delta^{*}(\mathrm{~mm})$ & $\theta(\mathrm{mm})$ & $H$ \\
\hline DEHS & 30 & 17.77 & 9.78 & 2.30 & 1.80 & 1.28 \\
HFSB & 30 & 18.90 & 10.47 & 2.50 & 1.94 & 1.28 \\
AFSB & 30 & 19.17 & 10.61 & 2.47 & 1.94 & 1.27 \\
1/7 power law & 30 & 16.33 & - & 2.04 & 1.59 & 1.29 \\
DEHS & 50 & 17.50 & 9.13 & 2.11 & 1.67 & 1.26 \\
HFSB & 50 & 18.68 & 9.97 & 2.28 & 1.81 & 1.26 \\
AFSB & 50 & 17.90 & 9.75 & 2.25 & 1.77 & 1.27 \\
1/7 power law & 50 & 14.75 & - & 1.84 & 1.43 & 1.29 \\
\hline
\end{tabular}

conducted with HFSB and even with the non-neutrally buoyant AFSB.

Measurements of the HFSB and AFSB diameter are again possible through the distance between the bubble glare points. The size distributions obtained during the boundary-layer measurements were very similar to those obtained upstream of the cylinder, meaning that the same production regimes were reproduced and the time responses estimated with the cylinder experiment can be assumed applicable to the measurements in the boundary layer. The diameter and standard deviation of each distribution and the normalized particle diameters $d_{p} / \delta^{*}$ and $d_{p} / \delta_{90}$ are summarized in Table 5. The bubble diameter is a significant fraction of the boundary-layer displacement thickness, resulting in data drop-out in the vicinity of the wall. Compared with the boundary-layer thickness $\left(\delta_{90}\right)$, the mean bubble diameter is two orders of magnitude smaller, indicating their potential ability to follow the most energetic turbulent fluctuations.

The Stokes numbers based on displacement thickness and the ratio of added-mass force to the Stokes drag are also shown in Table 5. The Stokes number for HFSB is about 0.5 , which should already raise a concern according to Samimy and Lele (1991) that have found a $2 \%$ error in the velocity measurement for $\mathrm{St}=0.2$. The latter work, however, was conducted for heavy small particles and some of the underlying hypotheses may need to be reconsidered for large neutrally buoyant tracers. Indeed, the ratio $F_{\mathrm{AM}} / F_{\mathrm{St}}$, which was calculated from (7) using $f=U_{\infty} / \delta^{*}$, indicates that the added-mass force is significantly important for both HFSB and AFSB in the turbulent boundary layer, while it is negligible for DEHS. The added-mass force is, therefore, expected to come into play and improve the tracing characteristics of these tracers when immersed in the turbulent boundary layer. In this case, the bubbles should follow the flow more accurately than it would have been presumed through analyzing solely the Stokes number.

The following results present the wall distance normalized with respect to the boundary-layer thickness $\delta_{90}$, based on the reference DEHS measurements. When referring to the boundary-layer edge, however, this is intended as $\delta_{99}$ $\left(\sim 1.8 \delta_{90}\right)$.

The number of detected particle images $n_{p}$ is illustrated in Fig. 9 normalized with the number of particles in the free stream $n_{p, \infty}$. The concentration in the outer region of the boundary layer is rather uniform. The mild increase from outside the boundary layer towards $y=0.5 \delta_{90}$ is ascribed to the Gaussian dispersion of bubbles from the single generation point centered approximately at the plate mid-plane. The cause of a peak concentration exhibited close to wall, instead, remains to be investigated, although it is suggested that it may be due to the integrated effect of shearing along the boundary layer.

Finally, the concentration of bubbles in the vicinity of the wall $\left(y<0.2 \delta_{90}\right)$ decreases dramatically, which is consistent with the hypothesis of bubbles bursting when they enter in contact with the wall.

The mean streamwise velocity profiles measured with the bubbles are in good agreement with the reference profile obtained by DEHS (Fig. 10). From the free stream, departures from the reference begin at $2 \delta_{90}$, from which the bubbles overall underestimate the mean velocity by
Table 5 Particle Stokes number. For DEHS, it is assumed that $d_{p}=1 \mu \mathrm{m}$ and $\tau_{p}=2 \mu \mathrm{s}$ (Ragni et al. 2011)

\begin{tabular}{llllllll}
\hline Particle & $U_{\infty}$ & $d_{p}(\mathrm{~mm})$ & $\sigma_{d}(\mathrm{~mm})$ & $d_{p} / \delta^{*}$ & $d_{p} / \delta_{90}$ & $S t_{\delta^{*}}$ & $F_{\mathrm{AM}} / F_{\mathrm{St}}$ \\
\hline HFSB & 30 & 0.55 & 0.06 & 0.25 & 0.06 & 0.4 & 46 \\
HFSB & 50 & 0.54 & 0.05 & 0.28 & 0.06 & 0.7 & 81 \\
AFSB & 30 & 0.42 & 0.03 & 0.19 & 0.04 & 5.8 & 27 \\
AFSB & 50 & 0.46 & 0.05 & 0.21 & 0.05 & 10.7 & 59 \\
DEHS & 30 & $\sim 10^{-3}$ & - & $4 \times 10^{-4}$ & $1 \times 10^{-4}$ & $3 \times 10^{-2}$ & $2 \times 10^{-4}$ \\
DEHS & 50 & $\sim 10^{-3}$ & - & $5 \times 10^{-4}$ & $1 \times 10^{-4}$ & $6 \times 10^{-2}$ & $3 \times 10^{-4}$ \\
\hline
\end{tabular}


approximately $2 \%$ of $U_{\infty}$ with differences between HFSB and AFSB in the same order.

The mean wall-normal velocity profiles are shown in Fig. 11. The shape of the curves and the magnitude of the mean wall-normal velocity are similar to what was found numerically by Sardina et al. (2012), with particles drifting

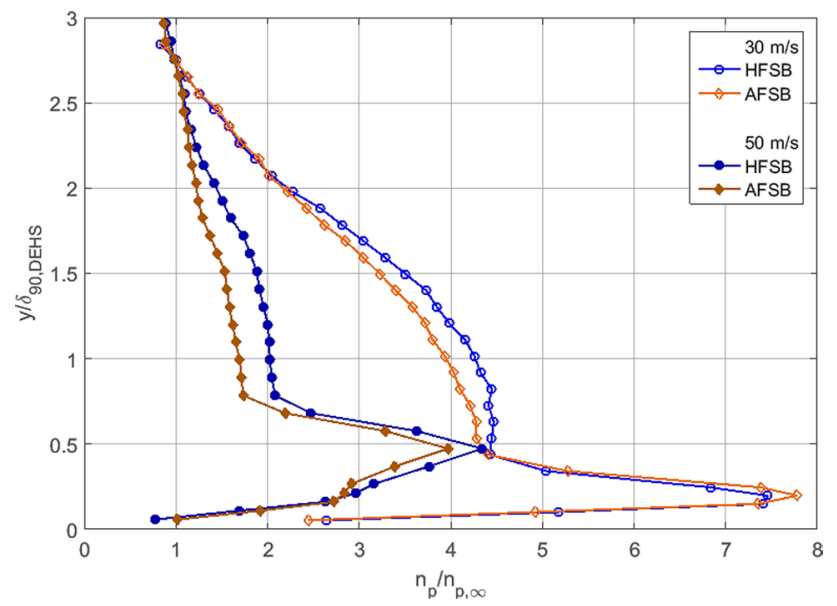

Fig. 9 Wall-normal distribution of the number of particles per unit area. Values normalized with respect to the free stream towards the wall along the boundary layer and away from it in the free stream. The DEHS particles show a slightly positive mean wall-normal velocity, consistent with the mechanism of boundary-layer growth. It should be remarked, however, that the vertical velocity component here is a small fraction of a percent with respect to the free stream value.

The normal Reynolds shear stress distributions are computed from the root mean square at every $(\mathrm{x}, \mathrm{y})$ grid position and illustrated in Fig. 12. The normal stresses appear to be measured in good agreement with the reference data. The distribution of $u_{\text {rms }}^{\prime}$ and $v^{\prime}$ rms for both HFSB and AFSB follows that of DEHS with discrepancy of about $0.5 \%$ of $U_{\infty}$. No systematic pattern can be observed for these deviations, indicating that errors are more of random type than systematic deviations. It is conjectured that the relatively accurate estimation of velocity fluctuations measured with AFSB in spite of the large relaxation time based on Stokes flow assumption is significantly corrected by the presence of the added-mass force (see Table 5). Thus, a simple estimation of the time response neglecting the unsteady forces (Eq. 3) most likely results in an underestimation of the cut-off frequency, above which the particles are unable to follow the flow. The lower values of turbulent fluctuations yielded by HFSB and AFSB at the edge and outside the boundary layer
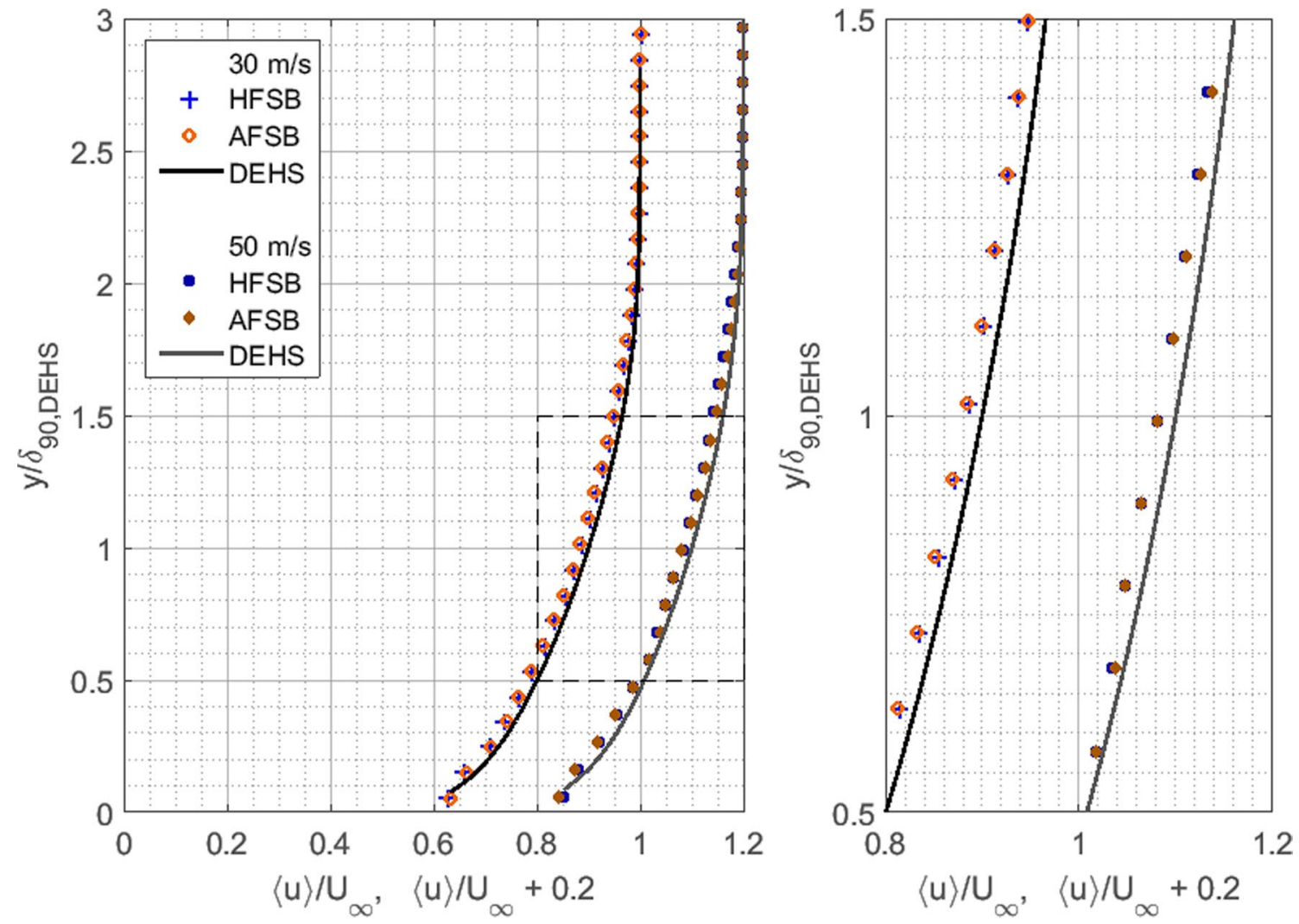

Fig. 10 Mean streamwise velocity profiles measured with HFSB and AFSB at 30 and $50 \mathrm{~m} / \mathrm{s}$ (shifted to the right by 0.2 ). The reference velocity measured with planar PIV and DEHS tracers is given (black solid line) 


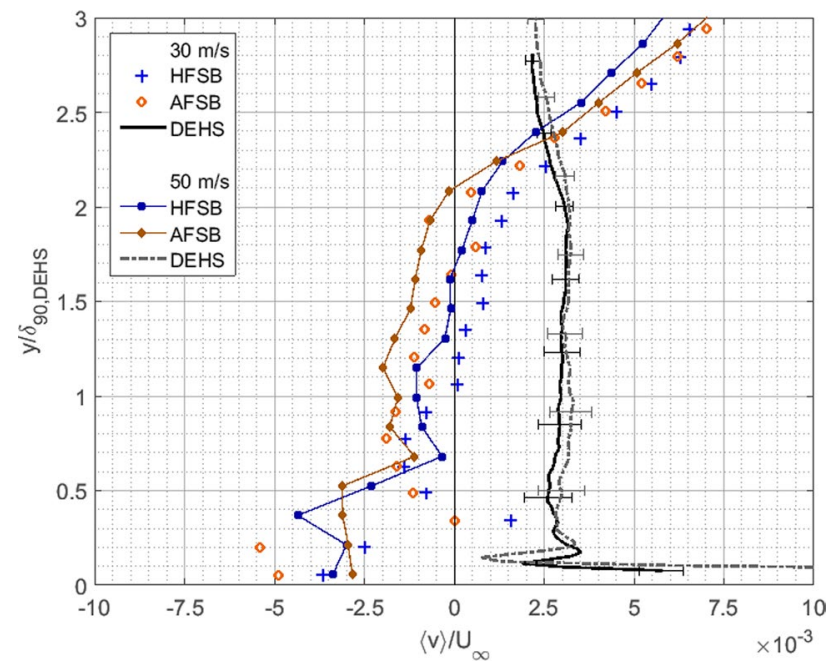

Fig. 11 Mean wall-normal velocity profiles measured with HFSB and AFSB at 30 and $50 \mathrm{~m} / \mathrm{s}$, compared with measurements performed with DEHS tracers

in comparison to the reference data are ascribed to the higher level of random errors associated to the measurements with DEHS. The latter make use of two-frame cross-correlation, which systematically overestimates the free stream turbulence intensity by approximately $0.5 \%$ of $U_{\infty}$ (considering a sub-pixel precision of 0.05 px, Raffel et al. 2007).

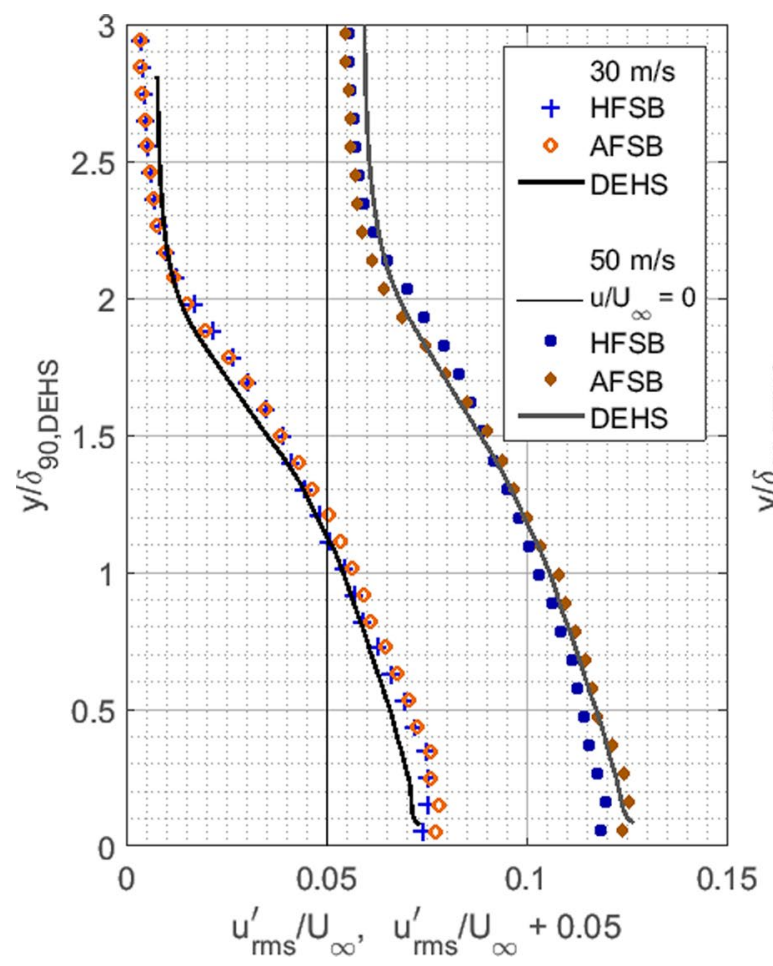

The normalized Reynolds shear stress profiles (Fig. 13) show a systematic discrepancy with respect to the reference data. The soap bubbles' measurements overestimate $u / v \prime$ in the region $y<0.8 \delta_{90}$. At free stream velocity of $30 \mathrm{~m} / \mathrm{s}$, the HFSB tracers exhibit a visible deviation from the reference of approximately $10 \%$ the local value, with a peak departure of $30 \%$ at $y=0.4 \delta_{90}$. At the same speed, the inertial particles have a more pronounced deviation overestimating the value of $R_{x y}$ by approximately $20 \%$ and a maximum difference of $50 \%$ at $y=0.15 \delta_{90}$. The cause of the above discrepancy, especially for the HFSB tracers, is not yet understood and should be subjected to further scrutiny.

At higher free stream velocity, the discrepancy between HFSB and the reference data is surprisingly small, with only a minor underestimation in the lowest portion of the boundary layer $\left(y<0.4 \delta_{90}\right)$. This behavior is somehow in contrast with the expectation that at higher free stream velocity, the measured discrepancy should be exacerbated by the higher level of fluid flow accelerations. The Reynolds shear stress peaks measured with AFSB are systematically overestimated also at $50 \mathrm{~m} / \mathrm{s}$, indicating that inertial effects dominate the error in $R_{x y}$. The departure to the reference data is in the order of $15 \%$ with peak value of $30 \%$ around the $y=0.5 \delta_{90}$.

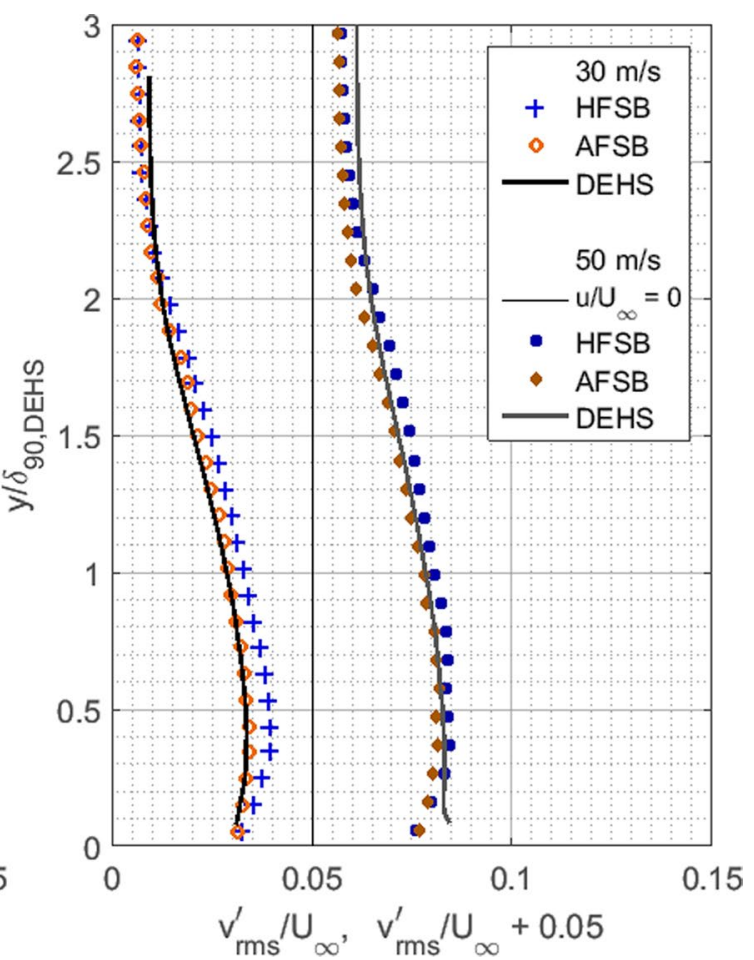

Fig. 12 Normalized root mean square of streamwise (left) and wall-normal (right) velocity fluctuations measured with DEHS, HFSB, and AFSB at 30 and $50 \mathrm{~m} / \mathrm{s}$. The $50 \mathrm{~m} / \mathrm{s}$ profiles are shifted to the right to ease visualization 
Fig. 13 Normalized Reynolds shear stress measured with DEHS, HFSB, and AFSB at 30 and $50 \mathrm{~m} / \mathrm{s}$. The profiles at $50 \mathrm{~m} / \mathrm{s}$ free stream velocity are shifted to the right to ease the visualization

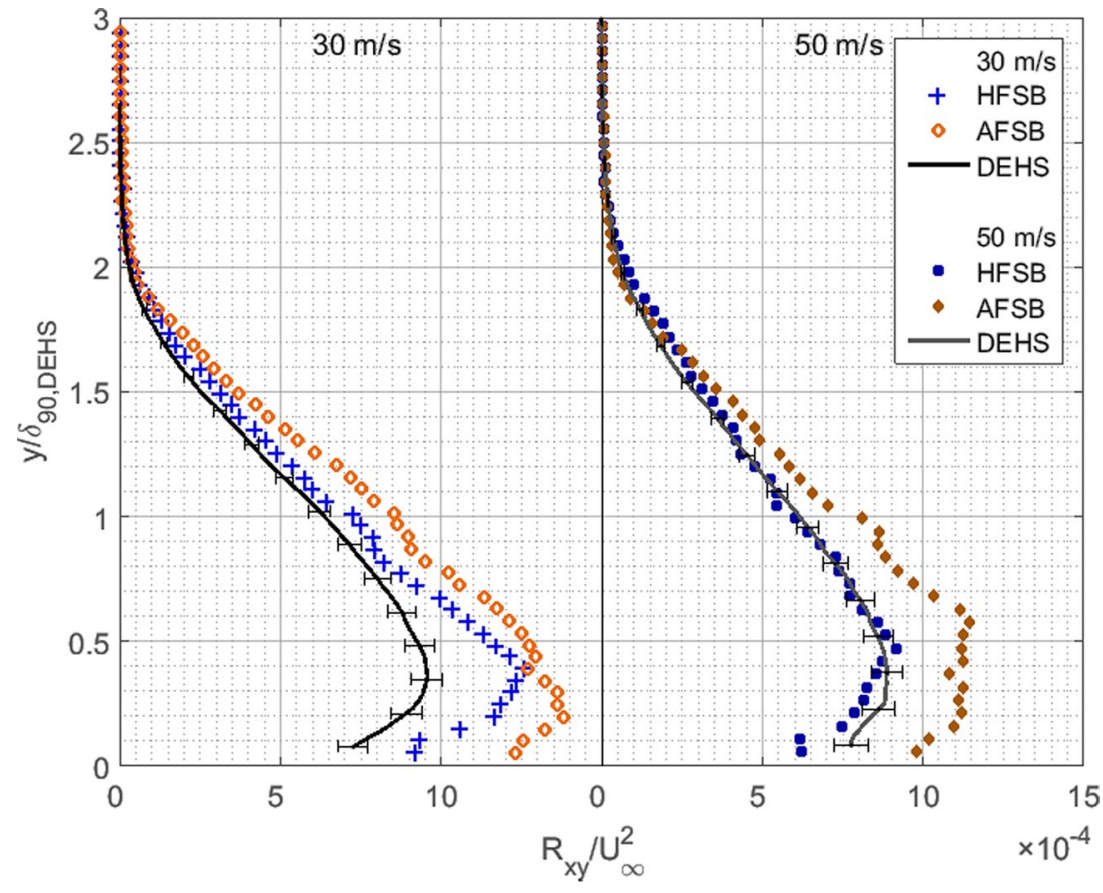

\section{Conclusions}

Neutrally buoyant helium-filled soap bubbles (HFSB) have been shown to enable accurate measurements of turbulent boundary-layer properties at $\operatorname{Re}_{\theta}$ in the order of $10^{3}$ to $10^{4}$, showing a good tracing fidelity in non-isotropic turbulence at moderate speeds.

The properties of the tracers used in the experiments have been carefully evaluated by means of an additional experiment that yields the distribution of their diameter and time response from which their density relative to the air is estimated. HFSB tracers have a rather monodisperse distribution and a measured reaction time of approximately $30 \mu \mathrm{s}$. The air-filled bubbles have a comparatively slower response approaching $0.5 \mathrm{~ms}$. The respective values of the Stokes number are in the order of 0.5 and 5 for HFSB and AFSB, indicating that they operate in a regime where tracing errors are expected, certainly for AFSB.

Compared to the conventional micron-sized tracers (DEHS), measurements using HFSB allow estimating the boundary-layer thickness within $10 \%$; the mean streamwise velocity profile deviated less than $2 \%$ of $U_{\infty}$ across the boundary layer down to a wall distance of approximately two bubble diameters. The turbulent fluctuations departed by less than $0.5 \% U_{\infty}$ from the reference. Larger differences were observed in the Reynolds shear stress profiles close to the wall of up to $30 \%$ of the peak value measured by DEHS for $30 \mathrm{~m} / \mathrm{s}$.

Although inertial air-filled soap bubbles (AFSB) are not able to follow streamline curvatures in the potential flow ahead of a cylinder, in the zero-pressure-gradient turbulent boundary layer, they seem to provide acceptable accuracy for the turbulence statistics, comparable to that obtained with neutrally buoyant tracers. The unsteady forces are hypothesized to be non-negligible for the bubbles in the turbulent boundary layer and the added-mass force appears to be particularly important. This is experimentally proven by the measurements of the Reynolds stresses, which show no visible modulation effect for AFSB in comparison to what observed in the flow ahead of the cylinder. On the other hand, the turbulent shear stress appears to be systematically and significantly overestimated by up to $50 \%$ of the peak value.

Finally, considering that the studied AFSB tracers are four times heavier than air, the observed deviations in the results indicate that the use of HFSB tracers that are typically only slightly lighter or heavier than air should not introduce significant tracing errors.

Open Access This article is distributed under the terms of the Creative Commons Attribution 4.0 International License (http://creativeco mmons.org/licenses/by/4.0/), which permits unrestricted use, distribution, and reproduction in any medium, provided you give appropriate credit to the original author(s) and the source, provide a link to the Creative Commons license, and indicate if changes were made.

\section{References}

Adrian RJ, Westerweel J (2011) Particle image velocimetry. Cambridge University Press, New York

Bosbach J, Kühn M, Wagner C (2009) Large scale particle image velocimetry with helium filled soap bubbles. Exp Fluids 46:539 
Bourgoin M, Qureshi NM, Baudet C, Cartellier A, Gagne Y (2011) Turbulent transport of finite sized material particles. J Phys Conf Ser 318:012005

Caridi GCA, Ragni D, Sciacchitano A, Scarano F (2016) HFSB seeding for large scale tomographic PIV in wind tunnels. Exp Fluids 57:190

Cierpka C, Lütke B, Kähler CJ (2013) Higher order multi-frame particle tracking velocimetry. Exp Fluids 54:1533

Clift R, Grace J, Webe M (1978) Bubbles, drops and particles. Academic Press, New York

Daitche A (2015) On the role of the history force for inertial particles in turbulence. J Fluid Mech 782:567-593

Dehaeck S, van Beeck JPAJ., Riethmuller ML (2005) Extended glare point velocimetry and sizing for bubbly flows. Exp Fluids 39:407-419

Kerho MF, Bragg MB (1994) Neutrally buoyant bubbles used as flow tracers in air. Exp Fluids 16:393

Kühn M, Ehrenfried K, Bosbach J, Wagner C (2011) Large-scale tomographic particle image velocimetry using helium-filled soap bubbles. Exp Fluids 50:929

Malik NA, Dracos T, Papantoniou DA (1993) Particle tracking velocimetry in three-dimensional flows. Exp Fluids 15:279

Maxey MR, Riley JJ (1983) Equation of motion for a small rigid sphere in a nonuniform flow. Phys Fluids 26(4):883

Mei R (1996) Velocity fidelity of flow tracer particles. Exp Fluids 22:1

Morias KLL, Caridi GCA, Sciacchitano A, Scarano F (2016) Statistical characterization of helium-filled soap bubbles tracing fidelity for PIV. In: 18th international symposium on the application of laser and imaging techniques to fluid mechanics, Lisbon
Okuno Y, Fukuda T, Miwate Y, Kobayashi T (1993) Development of three dimensional air flow measuring method using soap bubbles. JSAE Rev 14(4):50-55

Pounder E (1956) Parachute inflation process, Wind-Tunnel Study WADC Technical report 56-391, Equipment Laboratory of Wright-Patterson Air Force Base pp. 17-18, Ohio, USA

Raffel M, Willert CE, Wereley ST, Kompenhans J (2007) Particle image velocimetry - a practical guide, 2nd edn. Springer, Berlin

Ragni D, Schrijer F, van Oudheusden BW, Scarano F (2011) Particle tracer response across shocks measured by PIV. Exp Fluids 50:53

Samimy M, Lele SK (1991) Motion of particles with inertia in a compressible free shear layer. Phys Fluids A 3:1915-1923

Sardina G, Schlatter P, Picano F, Casciola CM, Brandt L, Henningson DS (2012) Self-similar transport of inertial particles in a turbulent boundary layer. J Fluid Mech 706:584-596

Scarano F, Ghaemi S, Caridi GCA, Bosbach J, Dierksheide U, Sciacchitano A (2015) On the use of helium filled soap bubbles for large scale tomographic PIV in wind tunnel experiments. Exp Fluids 56:42

Schlichting H (1979) Boundary-layer theory, 7th edn. McGraw-Hill Inc., New York

Schneiders JFG, Caridi GCA, Sciacchitano A, Scarano F (2016) Largescale volumetric pressure from tomographic PTV with HFSB tracers. Exp Fluids 57:164

Sciacchitano A, Wieneke B (2016) PIV uncertainty propagation. Measur Sci Technol 27(8):084006 